\title{
Relationships between body dimensions, body weight, age, gender, breed and echocardiographic dimensions in young endurance horses
}

\author{
D. S. Trachsel ${ }^{1,2^{*}}$, A. Giraudet ${ }^{2}$ D. Maso², G. Hervé ${ }^{2}$, D. D. Hauri ${ }^{3}$, E. Barrey ${ }^{4}$ and C. Robert ${ }^{2,4}$
}

\begin{abstract}
Background: The heart's physiological adaptation to aerobic training leads to an increase in heart chamber size, and is referred to as the Athlete's heart. However, heart dimensions are also related to body weight (BWT), body size, growth and (in some species) breed. There are few published data on the relationships between heart dimensions and growth or aerobic training in Arabian and Arabian-related endurance horses. Therefore the objective of the present study was to describe the influence of body dimensions (body length $(\mathrm{BL})$, thoracic circumference $(\mathrm{TC})$, withers height $(\mathrm{WH})$ ), BWT, age, gender, breed (purebred Arabians, part-bred Arabians, Anglo-Arabians, and Others) and the initiation of endurance training on echocardiographic measurements in competition-fit endurance horses aged 4 to 6 years.

Results: Most left atrial (LA) and left ventricular (LV) dimensions increased with age, whereas LA and LV functional indices did not. Although there was no gender difference for LV dimensions, females had larger LA dimensions. In terms of breed, Anglo-Arabians had the largest LV dimensions. Regression models indicated that the included explanatory factors had a weak influence on heart dimensions. Age, body dimensions, breed and gender showed the most consistent influence on LA dimensions, whereas BWT, breed and kilometres covered in competition showed the most consistent influence on LV dimensions.

Conclusion: The increase in echocardiographic dimensions with age indicates on-going growth in our population of 4 to 6 year-old horses. We also observed small changes associated with the initiation of endurance training. Morphometric dimensions had a greater influence on LA dimensions, whereas LV dimensions were also influenced (albeit weakly) by parameters associated with exercise intensity. These results may therefore reflect early adaptations linked to the initiation of endurance training.
\end{abstract}

Keywords: (3-10): equine, Echocardiography, Cardiovascular, Non-invasive cardiac assessment, Endurance, Body weight, Body dimensions, Gender, Age, Breed

\section{Background}

The Athlete's heart is a physiological adaptation to aerobic training and athletes (especially endurance athletes) have larger hearts and thus a larger stroke volume than nonendurance trained or sedentary humans $[1,2]$. The Athlete's heart has also been described in various types of racehorse

\footnotetext{
* Correspondence: dagmart@sund.ku.dk

${ }^{1}$ CIRALE-Hippolia, Médecine Sportive, RD 674, F-14430 Goustranville, France ${ }^{2}$ Université Paris-Est, Ecole Nationale Vétérinaire d'Alfort, 7 avenue du Général de Gaulle, F-94704 Maisons-Alfort, France

Full list of author information is available at the end of the article
}

and endurance horses [3-7] and has been linked to the appearance of heart murmurs albeit with little impact on performance $[6,8-10]$. Furthermore, a high $\mathrm{VO}_{2 \max }$ (an index of exercise capacity) in horses has been related to larger left ventricular (LV) dimensions and LV mass [11, 12]. Larger hearts have been associated with better performance in some studies, $[4,5,13]$ but not in others [14] and the discrepancy between these studies can certainly be explained by varying parameters used to assess performance. It is nevertheless the case that in most equestrian sport 
disciplines, horses that train more, race more, earn more or participate in elite competitions have larger hearts than their peers $[4,5,7,13]$.

However, other factors (such as body dimensions, body weight (BWT) $[5,6,15]$, breed $[16,17]$ and gender $[5,6,13])$ might influence the heart's dimensions. Some of the studies in this field included non-fully mature horses $[3,5,13]$, whereas others also included adult horses [6, 7]. Especially in young individuals, growth is an important matter, and age at maturity differs from one breed to another [18-21]. Furthermore, training methods and the physical demands of competition differ from one discipline to another. Thus, conclusions based on data from one breed and/ or discipline cannot necessarily be fully extrapolated to another breed and/or discipline. Hence, the primary objective of the present study was to describe the development of the heart in young Arabian-type horses aged between four and six years, an age at which endurance training is usually initiated. The second objective was to assess a possible association between external factors and the echocardiographic dimensions of the heart's chambers and great vessels in these horses.

\section{Methods}

Population

Between 2011 and 2014, echocardiographic measurements were recorded during field exercise tests and at the annual finals of the French National Championships for young endurance horses. The field tests were organised by the research group for all interested endurance horses owners four times a year. Only horses aged between 4 and 6 years at the time of presentation and with at least one purebred Arabian parent were included in the study. All horses were examined by the official veterinarians on site and had thus been authorised to race prior to enrolment in the study. Each horse was identified by its French national identification number and was included only once in our subsequent analyses. The study design was approved by the local institutional Ethical Committee (ComEthANSES/ENVA/UPEC, Maisons-Alfort, France; approval number: 12/07/11-1). All owners provided their written, informed consent to participation before the horses underwent any study procedures and were told that they could withdraw their horse from the study at any time.

For each horse, body length $(\mathrm{BL}$, in $\mathrm{cm}$ : the distance between the shoulder at the palpable portion of the greater tubercle and the palpable portion of the ischial tuberosity), thoracic circumference ( $\mathrm{TC}$, in $\mathrm{cm}$ : circumference measured at girth placement), withers height (WH, in $\mathrm{cm}$ : height at the highest point of the withers) were measured with a tape measure. The BWT (in $\mathrm{kg}$ ) was measured using a portable horse-weighing platform (Horse weigh ${ }^{\oplus}$ Tokyo model, Horse Weigh Ltd, Llandrindod Wells, UK, http:// www.horseweigh.com). The body surface area (BSA, in $\mathrm{m}^{2}$ ) [22] was calculated using the following equation: BSW= $0.101 \times \mathrm{BWT}^{2 / 3}$. Horses were divided into age groups: Group $4 y$ included all 4-year old horses, Group $5 y$ included all 5-year old horses, and Group $6 y$ included all 6-year old horses. Each horse's gender and breed were also recorded. Although all the horses necessarily had a least one purebred Arabian parent (given the study's inclusion criterion), they were divided into four breed groups for further analysis: Group purebred Arabians comprised all the purebred Arabians and the Shagya Arabians, Group part-bred Arabians comprised crossbreeds between Arabians and Warmbloods, Group Anglo-Arabians comprised AngloArabians or Anglo-Arabian crossbreed, and lastly, Group Others comprised all other Arabian crossbreeds.

Given that the owners of the included horses applied a very broad variety of training programmes, it was not possible to objectively quantify the horses' training history. However, a rough estimation of each horse's activity prior to echocardiography was derived by noting the total number of kilometres covered in competition since the first registered competition ( $\mathrm{Km}$-career) and the numbers of days since the first registered competition (d-career) from the records in the French Equestrian Federation's database (FFECompet https://ffecompet.ffe.com/cheval [23]).

\section{Echocardiographic measurements}

Transthoracic, two-dimensional echocardiography (2DE) was performed on resting horses before they participated in the endurance competitions or field exercise tests. The echocardiography was performed by two experienced clinicians (AG for the 68 examinations in 2011, and DST for the 272 examinations in 2012-2014) using a portable ultrasound system (Vivid I, General Electric Healthcare Europe $\mathrm{GMBH}$, France) that enabled synchronous recording of the electrocardiogram (ECG). The field conditions and the limited time available for the examinations precluded the realisation of Doppler ultrasound examinations and allowed only recordings from the right parasternal long axis views, as described elsewhere in detail [24-27].

The measurements were made under supervision of the experienced clinicians by two well-trained persons independent of those who examined the horses (DM, $\mathrm{GH})$. To summarise, the dimension of the aortic (sinus) diameter (AOD), and the maximal pulmonary artery diameter (PAD) were measured at end-diastole on the right parasternal long-axis $2 \mathrm{DE}$ views of the left and right ventricular outflow tract, respectively. The atrial dimensions were assessed in a right parasternal longaxis $2 \mathrm{DE}$ view centred on the LA by measuring the maximal LA diameter $\left(\mathrm{LAD}_{\max }\right)$, and $\mathrm{LA}$ area $\left(\mathrm{LAA}_{\max }\right)$ at end-systole prior to mitral valve opening, the LA 
diameter $\left(\mathrm{LAD}_{\mathrm{a}}\right)$ and LA area $\left(\mathrm{LAA}_{\mathrm{a}}\right)$ at the onset of the electrocardiographic $\mathrm{P}$ wave prior to active contraction, and the minimal LA diameter $\left(\mathrm{LAD}_{\min }\right)$ and $\mathrm{LA}$ area $\left(\mathrm{LAA}_{\min }\right)$ at the time of mitral valve closure. The LV dimensions were also assessed in a right parasternal longaxis $2 \mathrm{DE}$ view but centred to visualize the LV apex. From this view, the interventricular septal thickness (IVS), the LV internal diameter (LVID), and the LV free wall thickness (LVFW) were measured on a line midway between the papillary muscles and the mitral valve annulus, perpendicular to the IVS and LVFW and parallel to the mitral valve annulus at end-diastole (d) and peak systole (s). Using the same view LV internal area (LVIA) and LV internal length (LVIL) were measured at end-diastole (d) and at peak systole (s).

The LA areas were used to calculate functional indices of LA contraction including the active LA fractional area change [Active LA FAC $=\left(\mathrm{LAA}_{\mathrm{a}}-\mathrm{LAA}_{\min }\right) / \mathrm{LAA}_{\mathrm{a}} \times$ 100], the passive LA fractional area change [Passive LA $\left.\mathrm{FAC}=\left(\mathrm{LAA}_{\max }-\mathrm{LAA}_{\mathrm{a}}\right) / \mathrm{LAA}_{\max } \times 100\right]$, the total LA fractional area change [Total LA FAC $=\left(\mathrm{LAA}_{\max }-\right.$ $\left.\left.\mathrm{LAA}_{\text {min }}\right) / \mathrm{LAA}_{\max } \times 100\right]$, and the active-to-total LA area change [active:total LA AC $=\left(\mathrm{LAA}_{\mathrm{a}}-\mathrm{LAA}_{\min }\right) /\left(\mathrm{LAA}_{\max }\right.$ - $\left.\left.\mathrm{LAA}_{\min }\right)\right]$ [25].

For measured LV dimensions, the relative wall thickness at end-diastole [RWT $=\left(\mathrm{IVS}_{\mathrm{d}}+\mathrm{LVFW}_{\mathrm{d}}\right) / \mathrm{LVID}_{\mathrm{d}}$, the mean wall thickness at end-diastole $\left[\mathrm{MWT}=\left(\mathrm{IVS}_{\mathrm{d}}\right.\right.$ $\left.\left.+\mathrm{LVFW}_{\mathrm{d}}\right) / 2\right]$ and the $\mathrm{LV}_{\text {mass }}\left[1.04 \times\left[\left(\mathrm{LVID}_{\mathrm{d}}+\mathrm{LVFW}_{\mathrm{d}}+\right.\right.\right.$ $\left.\mathrm{IVS}_{\mathrm{d}}\right)^{3}-$ LVID $\left.\left._{\mathrm{d}}\right]-13.6\right]$ were calculated.[5, 11, 24]. Two functional indices for the LV were calculated: the LV fractional shortening $\left[\mathrm{LV} F S=\left(\mathrm{LVID}_{\mathrm{d}}-\mathrm{LVID}_{\mathrm{s}}\right) / \mathrm{LVID}_{\mathrm{d}} \times\right.$ 100]) and the LV fractional area change [LV FAC $\left.=\left(\mathrm{LVIA}_{\mathrm{d}}-\mathrm{LVIA}_{\mathrm{s}}\right) / \mathrm{LVIA}_{\mathrm{d}} \times 100\right][5,11,24,27]$.

Further, the cardiac output $[\mathrm{CO}=\mathrm{SV} \times \mathrm{HR}]$ and the stroke volume $\left[\mathrm{SV}=\mathrm{LVIV}_{\mathrm{d}}-\mathrm{LVIV}_{\mathrm{s}}\right]$, based on $\mathrm{LV}$ internal volume at end-diastole $\left(\operatorname{LVIV}_{\mathrm{d}}\right)$ and at peak systole $\left(\operatorname{LVIV}_{\mathrm{s}}\right)$, were calculated using the equation 5/ $6 \times$ LVIA $^{2} /$ LVIL (describes as the "4CAL equation" by McConachie et al. [28]).

In order to compare the relative proportions of the heart's chambers, the $\mathrm{LAA}_{\max } / \mathrm{LVIA}_{\mathrm{d}}$, and $\mathrm{LAD}_{\max } /$ $\mathrm{LVID}_{\mathrm{d}}$ ratios were calculated. Further, the AOD/PAD ratio was established.

All measurements were made offline on the basis of digitally stored cine-loop recordings and were averaged over two or three non-consecutive cardiac cycles. The heart rate (HR) was determined and the rhythm was assessed on the ECGs recorded synchronously on the cine-loops.

\section{Statistical analyses}

Based on a visual assessment of histograms and of normal probability plots, normality of the data distribution was given and parametric methods were applied.
Influences of age category, gender and breed were tested using an analysis of variance (ANOVA) with Holm-Sidak post-hoc tests for multiple comparisons. Further, pooled males (geldings and intact males) were compared with females using a Student's $t$-test. Dichotomous variables were compared in a $\mathrm{Chi}^{2}$ test. The threshold for statistical significance was set to $p<0.05$.

Univariate regression models were established for LA and LV echocardiographic measurements and for explanatory factors with continuous data (BWT, BSA, $\mathrm{WH}, \mathrm{TC}, \mathrm{BL}, \mathrm{Km}$-career, d-career). Multivariate regression models were then developed for the most relevant LA and LV measurements. Based on an assessment of the residuals, log-linear regression models were used if necessary. The log transformation of the outcomes allowed reaching normality of the residuals. All explanatory factors were included in the initial model. Thereafter, quadratic and cubic terms were also included for the BWT in the initial models. Nonrelevant explanatory factors were excluded by stepwise elimination based on the adjusted $\mathrm{R}^{2}$ and the Akaike information criterion (AIC). However, models were not considered to be relevant if the final adjusted $R^{2}$ was below $10 \%$. No interactions between explanatory factors were considered in the models.

All statistical analyses were performed using commercially available software (GraphPad Prism ${ }^{\circ}$, version 6.05, GraphPad Software, San Diego, CA, USA, www.graphpad.com, SigmaStat ${ }^{\circ}$, version 3.5, Systat Software GmbH, Erkrath, Germany, R-software ${ }^{\circ}$, version 3.1.0, $\mathrm{R}$ Development Core Team, Vienna, Austria).

\section{Results}

Three hundred and forty horses were included in the study. The demographic characteristics of the study population are summarised in Table 1. Given that the field conditions limited the available time per horse, the number of missing data varied from one measurement to another. The numbers of available data per measurement are given in Additional files 1, 2, 3 and 4. Most horses were females or geldings, and intact males were significantly underrepresented $\left(\mathrm{Chi}^{2} p<0.01\right)$. Furthermore, most of the horses were purebred or part-bred Arabians, whereas Anglo-Arabians and Others were significantly underrepresented $\left(\mathrm{Chi}^{2} \quad p=0.03\right)$. There were slightly more horses in Group $6 y$ than in the two other groups. The measured HR was slightly higher than the usual resting HR for horses, and no arrhythmias were observed on the reviewed cine-loops.

The p-values obtained in ANOVAs for age groups, genders and breed groups are presented in Additional files 5, 6 and 7. In summary, the BWT and the BSA were significantly greater in 5-year old horses than in 4-year 
Table 1 Demographic characteristics of the study population

\begin{tabular}{|c|c|c|c|c|c|}
\hline & 4-year-olds & 5-year-olds & 6-year-olds & Total & $p^{\#}$ \\
\hline \multirow[t]{2}{*}{ Number } & 101 & 109 & 130 & 340 & n.s \\
\hline & Mean $( \pm S D)$ & Mean $( \pm S D)$ & Mean $( \pm S D)$ & Mean $( \pm S D)$ & \\
\hline $\mathrm{HR}(\mathrm{bpm})$ & $44( \pm 10)$ & $44( \pm 9)$ & $43( \pm 10)$ & $43( \pm 9)$ & n.s \\
\hline BWT (kg) & $405.9^{\mathrm{a}}( \pm 39.2)$ & $422.9^{b}( \pm 35.3)$ & $411.1^{\mathrm{a}}( \pm 37.0)$ & $413.4( \pm 37.6)$ & * \\
\hline BSA $\left(m^{2}\right)$ & $3.9^{\mathrm{a}}( \pm 0.2)$ & $4.0^{\mathrm{b}}( \pm 0.2)$ & $3.9^{\mathrm{a}}( \pm 0.2)$ & $3.9( \pm 0.2)$ & * \\
\hline $\mathrm{WH}(\mathrm{cm})$ & $152.9( \pm 3.8)$ & $153.5( \pm 4.0)$ & $152.5( \pm 4.3)$ & $152.9( \pm 4.0)$ & n.s. \\
\hline $\mathrm{TC}(\mathrm{cm})$ & $173.5( \pm 7.6)$ & $174.7( \pm 6.8)$ & $173.1( \pm 5.8)$ & $173.8( \pm 6.7)$ & n.s. \\
\hline $\mathrm{BL}(\mathrm{cm})$ & $154.2( \pm 7.3)$ & $154.7( \pm 7.5)$ & $152.9( \pm 7.4)$ & $153.9( \pm 7.4)$ & n.s. \\
\hline d-career (days) & $109.7^{\mathrm{a}}( \pm 69.5)$ & $302.5^{\mathrm{b}}( \pm 175.1)$ & $576.3^{c}( \pm 268.8)$ & $351.5( \pm 276.5)$ & $* *$ \\
\hline Km-career $(\mathrm{km})$ & $44.3^{\mathrm{a}}( \pm 49.7)$ & $130.5^{\mathrm{b}}( \pm 82.8)$ & $435.8^{c}( \pm 323.6)$ & $223.0( \pm 269.8)$ & $* *$ \\
\hline \multicolumn{6}{|l|}{ Gender } \\
\hline females & 42 & 52 & 64 & 158 & $* *$ \\
\hline geldings & 34 & 36 & 48 & 118 & \\
\hline intact males & 25 & 21 & 18 & 64 & \\
\hline \multicolumn{6}{|l|}{ Breed } \\
\hline Purebred Arabian & 49 & 64 & 88 & 201 & * \\
\hline Part-bred Arabians & 39 & 30 & 31 & 100 & \\
\hline Anglo-Arabians & 5 & 11 & 8 & 24 & \\
\hline Others & 8 & 4 & 3 & 15 & \\
\hline
\end{tabular}

\#, results from ANOVAs or $\mathrm{Chi}^{2}$ tests, as appropriate; ${ }^{*}$, significant at $p<0.05 ;{ }^{* *}$, significant at $p<0.01$; n.s., not significant; results marked with a different superscript letter differed significant from the other results in the row. For $p$-values, see Additional file 5

or 6-year old horses. The body dimensions in Group $5 y$ were also slightly (but not significantly) higher than those in the other two age groups.

Further, the horses in Group $6 y$ had significantly longer careers and had covered significantly more kilometres in competition than those in the other age groups. Likewise, values for $\mathrm{Km}$-career and $\mathrm{d}$-career were also significantly higher in Group $5 y$ than in Group $4 y$.

The two-dimensional LA dimensions increased fairly steadily with age and significant differences were noted when comparing Group $4 y$ with Group 5y or Group $4 y$ with Group 6y (Additional file 6). However, the largest difference was observed when comparing Group $4 y$ and Group 6y. LA area measurements also increased with age, although the differences between the age groups were not significant. The LA functional indexes did not differ significantly with age. Group $5 y$ and Group $6 y$ did not differ significantly in terms of any of the LA dimensions.

AOD and AOD/PAD increased constantly from Group $4 y$ to Group 6y, whereas PAD was lower in Group $4 y$ than in Group 5y (Additional file 6).

Most LV echocardiographic measurements (Additional file 7) also increased with age and the largest differences were observed when comparing Group $4 y$ and $5 y$. The differences between Group $5 y$ and $6 y$ were less pronounced but were most marked for two-dimensional measurements. In contrast, increases in area measurements were more regular over the studied age range. Group $5 y$ and $6 y$ did not differ significantly in terms of any of the LV measurements. We did not observe any age-related changes for IVS $, I_{\mathrm{s}}, \mathrm{LVFW}_{\mathrm{s}}, \mathrm{LVFW}_{\mathrm{d}}$, MWR, RWT, LV FS, LV FAC and CO.

Overall, gender differences were small (Additional files 5,6 and 7). The comparison between genders showed that females had a larger TC than intact males or pooled males. Geldings had slightly greater WH and BWT than the other groups, and intact males had the lowest values for all morphometric dimensions; however, the differences were not statistically significant. There were no gender differences in $\mathrm{Km}$-career or $\mathrm{d}$-career. Intact males had the smallest two-dimensional LA dimensions, whereas females had the largest LA area measurements (Additional file 6). These differences in dimensions led to a significant gender difference for one of the LA functional indices: the passive LA FAC was highest in intact males. There were no significant differences for other comparisons. With regard to the great vessels, the AOD was slightly (but not significantly) lower in females. The PAD was significantly greater in females when compared with geldings or with all males. Hence, the ratio AOD/PAD was also smaller in females than in geldings or in all males. There were no gender differences in the LV measurements (Additional file 7). 
There were no differences for $\mathrm{Km}$-career or d-career between the breed groups. In our population purebred Arabians were smallest and lightest, whereas AngloArabians were tallest and heaviest (Additional file 5). Further, none of the differences in LA dimensions between breed groups were statistically significant (Additional file 6). However, two-dimensional LA measurements were highest in Anglo-Arabians, whereas in partbred Arabians and Others these dimensions were slightly smaller than in purebred Arabians. The same difference was not apparent for area measurements, and purebred Arabians even showed a trend towards highest values. There were no obvious differences in AOD or in PAD between the breeds.

As seen for the LA, all LV measurements were highest in Anglo-Arabians and for most measurements lowest in part-bred Arabians and Others (Additional file 7). The largest differences were observed for measurements made at end-diastole, and were statistically significant for $\mathrm{IVS}_{\mathrm{d}}, \mathrm{LVID}_{\mathrm{d}}, \mathrm{LV}_{\text {mass }}$ and SV.

Univariate linear regression models revealed very weak influences of the explanatory variables on echocardiographic measurements (Additional files 1 and 2). Less than $14 \%$ of the total variability in the LV dimensions $\left(\right.$ LVIA $_{d}: R^{2}=11.0 \% ;$ LVIV $_{d}: R^{2}=12.4 \%$;V: $\left.R^{2}=12.6 \%\right)$ and in the $L_{\text {mass }}\left(R^{2}=13.7 \%\right)$ was explained by the BWT or BSA. Less than $11 \%$ of the total variability of the LA measurements was explained by $\mathrm{TC}\left(\mathrm{LAD}_{\mathrm{a}}: \mathrm{R}^{2}=\right.$ $11.0 \%$; $\mathrm{LAA}_{\max }: \mathrm{R}^{2}=11.0 \%$ ).

In the multivariate regression models, the values for the adjusted $\mathrm{R}^{2}$ were also low and the chosen explanatory factors accounted for less than $18 \%$ of the total variability in the echocardiographic measurements (Additional files 3 and 4). Furthermore, the analyses for LA and LV measurements showed different patterns. For LA measurements, the values for the adjusted $\mathrm{R}^{2}$ were higher than those obtained for LV measurements, and most of the LA measurements were related to morphometric measurements.

In these multivariate regression models, BL showed a positive influence on $\mathrm{LAD}_{\max }, \mathrm{BL}$ and $\mathrm{TP}$ showed a positive influence on $\mathrm{LAD}_{\mathrm{a}}$ and $A O D$. BL and $\mathrm{WH}$ had a positive influence on $\mathrm{LAD}_{\text {min }}$. $\mathrm{LAA}_{\mathrm{a}}$ and $\mathrm{LAA}_{\text {min }}$ increased with increasing $\mathrm{WH}, \mathrm{TP}$ and $\mathrm{BL}$, whereas $\mathrm{LAA}_{\max }$ increased only with increasing TP and WH. BWT had positive influence only on $\mathrm{LAD}_{\max }$, AOD and PAD. BSA is not reported as results were identical to those for BWT.

For most LA measurements, age positively influenced the dimensions. Hence older horses displayed larger dimensions, except for the PAD, which decreased with age.

Although Anglo-Arabians showed a trend towards larger two-dimensional LA measurements, breed had a negative influence on $\mathrm{LAD}_{\text {max }}, \mathrm{LAA}_{\mathrm{a}}$ and $\mathrm{LAA}_{\text {min }}$ in the multivariate regression models and belonging to a breed other than purebred Arabians was associated with a decrease in the mentioned LA dimensions.

As seen in the ANOVAs, multivariate regressions showed that gender was variously associated with the magnitude of some LA measurements. Intact males and geldings showed lower $\mathrm{LAD}_{\mathrm{a}}, \mathrm{LAD}_{\text {min }}, \mathrm{LAA}_{\mathrm{a}}$ and $\mathrm{LAA}_{\text {min }}$ values than females did. Intact males and geldings had smaller PADs but higher AODs than females. The Kmcareer and d-career variables were not associated with the LA measurements. The PAD increased with $\mathrm{Km}$-career and decreased very slightly with d-career.

For LV measurements (Additional file 4), most measurements were positively associated with BWT. This was especially true for $L V_{\text {mass }}, L_{V} A_{d}$, and derived values such as $\mathrm{LVIV}_{\mathrm{d}}$ and $\mathrm{SV}$. When considering other morphometric measurements, WH had positive influence on LVIA $_{d}, \operatorname{LVIV}_{d}$ and SV, and BL had a negative influence on LVIA $_{d}, \operatorname{LVIV}_{d}$ and SV. The results for BSA are not reported in detail because they were identical to those for BWT.

In comparison to purebred Arabians, $\mathrm{LV}_{\text {mass }}$ was higher in the Anglo-Arabians and lower in the part-bred Arabians. Gender and age had only a slight influence on LV measurements. However, $\mathrm{Km}$-career was positively associated with $\mathrm{LV}_{\text {mass }}, \mathrm{LVIA}_{\mathrm{d}}$ and $\mathrm{LVIV}_{\mathrm{d}}$.

\section{Discussion}

The present study reports changes in echocardiographic dimensions of the heart in young Arabian type horses at the end of their growth period and at the beginning of their endurance training for competitions. All external variables included in the analyses influenced to some extent several of the measured dimensions. Morphometric dimensions, age and breed showed most consistently an influence on LA measurements, whereas BWT, breed and Km-career had most consistently an influence on LV measurements.

All the horses in the study population had at least one purebred Arabian parent. Our study population was chosen to be representative of the horses participating in endurance competitions. This choice was in accordance with literature data indicating that purebred or mixedbreed Arabians are largely overrepresented in endurance competitions, although some other breeds do participate $[29,30]$. In view of the inclusion criteria, the division into several breed groups may seem arbitrary. However, there were significant differences between these breed groups. Purebred Arabians were the smallest and lightest - giving them advantage over other breeds in endurance competitions $[29,30]$. Body dimension and BWT were highest in Anglo-Arabians that in turn led also to larger $\mathrm{LV}_{\text {mass. }}$ Nevertheless, in the multivariate regression models, belonging to breeds other than purebred Arabians was associated with smaller LA measurements. This 
indicated that the smallest horses in our population did not necessarily have the smallest hearts and hence that other factors must be considered.

BWT and body dimensions were higher in Group 5y than in Group 4y, which was presumably due to on-going growth. In contrast, the smaller body dimensions in Group $6 y$ (especially $\mathrm{WH}$ and $\mathrm{BL}$, which are less influenced by body condition) might be due to the selection of smaller, lighter, more compact horses for more demanding competitions. Indeed, high BWT in endurance horses has been associated with failure to finish competitions, particularly due to lameness [29, 30]. Furthermore, horses in Group $6 y$ had covered a greater distance in competition ( $\mathrm{Km}$-career) and thus were certainly subjected to harder training, which may have resulted in a lower BWT. Training reduces fat content and increases muscle mass. Therefore, it would have been interesting to measure lean body weight. BWT and especially fat-free mass were associated with better indices of performances in Standarbreds, [31, 32] and fat-free mass was also associated with a higher $\mathrm{VO}_{2 \max }$. However, too low BWT or too low percentage of body fat is detrimental in human athletes [33, 34]. The optimal percentage of body fat has not yet been established for horses, although body fat was around $8 \%$ in well-performing athletic horses [29, 32, 35]. As is the case for human athletes, the optimal percentage of body fat doubtless varies from one discipline to another $[34,36]$.

All heart dimensions increased with age. The difference between 4 and 5 years of age was greater than the difference between 5 and 6 years of age. In univariate and multivariate regression analyses, for most heart dimensions a weak relationship was observed with BWT or TC (especially for LA measurements). The higher values for the $\mathrm{R}^{2}$ in regression models of LA measurements and of the dimensions of the great vessels, and their relationship with body dimensions (rather than BWT) might indicate that these dimensions are more strongly related to the horse's morphotype. AlHaidir et al. also have reported that some echocardiographic measurements are somewhat more strongly associated with TC than they are with BWT [37]. However, due to the present study's inclusion criteria, the range of body dimensions and weights was narrower than in the work by $\mathrm{Al}$ Haidir et al. [37]. Concomitant increases in all dimensions are seen with growth, and growth has to be taken into consideration when studying young individuals. Different breeds have differing growth rates. Thoroughbreds and Standardbreds are fast-growing breeds and reached $83 \%$ of the adult BWT by the age of 2 years, whereas the growth rate is slower in other breeds and by the age of 2 years Warmbloods, Anglo-Arabians and Lusitanos reached $80 \%$, $75 \%$ and $77 \%$ of their adult BWT, respectively [20, 21]. Arabians have been classified as slow-growing horses [38]. Accordingly, they are still growing between the ages of 4 and 6 years. This contrasts with Standardbred horses, which are considered to reach maturity between the ages of 3.5 and 5 years [6]. Although growth certainly affects the LV dimensions, the latter are also greatly modulated by other factors. In particular, training is a strong stimulus for cardiac hypertrophy, and we detected a weak but significant association between $\mathrm{Km}$-career and some of the LV measurements. This very weak association might be due to the poorly elaborated performance parameters $(\mathrm{Km}$-career, $\mathrm{d}$ career) and also due to the generally low level of endurance training seen at this age [39]. A training-related increase in LV dimensions has been observed in Thoroughbreds [3], Standardbreds [5, 6] and elite Arabians [7]. The absence of a change in RWT indicated that the heart's adaptation to exercise in our population is more similar to that seen in Standardbreds $[5,6]$ than to that seen in Thoroughbreds, [3] or elite Arabians [7]. Further, this finding might also illustrate differences in training practices among different breeds. We did not find any association between LA dimensions and $\mathrm{Km}$-carrer. This is in contrast to studies in human athletes that showed also greater LA dimensions in presence of an Athlete's heart [40, 41]. However, the endurance training in our study population was probably not intensive enough to induce changes in LA dimensions.

The results of our regression analyses agree with another study reporting small values for the $R^{2}$ for $L V$ measurements in young (2-year-old) Standardbreds [6]. However, values for the $R^{2}$ increased with age in this previous study, particularly for $\mathrm{LV}_{\text {mass. }}$. This comparison also suggests that our population's training was not yet hard enough to induce marked changes in heart dimensions.

In accordance with previous studies [42-44], there were no age-related differences in LA and LV functional indexes.

Females had the largest TC. Given that LA measurements were related to TC in our regression models, we were not surprised to find that females had also the largest LA measurements. On the contrary, intact males had the smallest LA dimensions. In contrast to other studies [5, 13,44], gender did not appear to influence LV dimensions in our study population. One explanation could be that the intensity of the aerobic training in endurance horses at this age is not high enough to bring out the full potential for hypertrophy in intact males. Another explanation might be related to the custom of French endurance trainers/owners to breed females at the age of 5 years. Gestation may have induced changes in heart dimensions (as seen in humans and dogs), although these changes are usually reversible $[45,46]$.

The present study had a number of limitations. Due to field conditions and the limited time available per horse, not all echocardiographic views of heart chambers could be taken, and some views were of poor quality. Similarly, 
it was not possible to perform Doppler examinations and thus screen for possible regurgitations. Due to these technical issues there were several missing data. Nevertheless our analyses were based on a large body of data. Given that the measured heart chamber dimensions can be influenced by the transducer position and the views obtained [47, 48], not all of our measurements can be validly compared with the literature values. Our comparison with previous studies is therefore limited to the consideration of factors that influence heart dimensions. Second, the use of only longitudinal views meant that our LV measures could probably be underestimated [47]. Furthermore, the measured HRs were slightly over resting values, and the shorter filling time at these HRs might have affected ours results, especially for diastolic measurements. Nevertheless, the HRs measured in our studies were only slightly higher than those reported in the literature for endurance horses $[7,48]$. Lastly, the fact that the recordings and measurements were made by several operators probably decreased the precision of our measurements $[25,49]$.

Differentiating the effects of growth from the effects of training would have required the use of a control group. In principle, a control group should comprise untrained, non-competing horses of the same age. Unfortunately, this type of population was not available in our research setting. Furthermore, follow-up measurements of our study population 3-4 years later or measurements from a control group of mature, elite endurance horses would have provided information on the effects of more intensive aerobic training. In accordance with results from Sleeper et al. [7] reporting greater LVID, $\mathrm{LV}_{\text {mass }}$ and SV in elite Arabians (mean age 21.1, age range 7-17) than in non-elite Arabians, data from our research group show that in a smaller group of endurance horses ( $n$ =11; nine purebred Arabians and two others; four females, six geldings and one intact male; mean age ten, range 8-13 years) participating in races of least $120 \mathrm{~km}$, the main differences relative to younger horses were seen for LVID, LVIA, LVIL and LVIV, with greater differences appearing in peak systolic measurements. Furthermore, in the older horses, $L_{\text {mass }}$ was higher and LV FAC and LV FS were lower. The greater $L V$ dimensions and $L V_{\text {mass }}$ agree with literature data on various breeds $[4,6,7]$ and are certainly related to a greater aerobic training volume at this age. These considerations strengthen our hypothesis whereby the training load in younger horses was not intense enough to induce marked cardiac hypertrophy. Our findings with regard to LV FAC and LV FS are more difficult to explain, since the Athlete's heart does not feature any changes in LV function at rest $[1,41]$. The results might be best explained by the fact that older horses were more used to being handled and therefore showed a narrower range of heart rates than younger horses. Indeed, the HRs for adult endurance horses reported in the literature were slightly lower than in the present study $[7,48]$.

Furthermore, the performance parameters used in the present study ( $\mathrm{Km}$-career, $\mathrm{d}$-career) were very rough. In France, purebred Arabians are not trained intensively between the ages of 4 and 6 years and training is not as standardised as it is in Thoroughbreds or Standardbreds [39]. Furthermore, some horses were bred during this period, which decreased the training period. Therefore, it was not possible to analyse objective parameters, such as gains, speed at kilometre, or numbers of start $[4,5,13,14]$. The most accessible parameters were $\mathrm{Km}$-career and $\mathrm{d}$-career, since this information was freely available in the FEE database. We hypothesised that a horse having participated in a greater number of competitions or longer competitions would have been trained harder. Even though we observed a weak relationship between $\mathrm{Km}$-career and some LV measurements, better performance indicators are clearly needed. Long-term information on the endurance horses' competitive performance was not yet available at the time of the present study and will be addressed in the future.

Lastly, we had no possibility to record and therefore to compare echocardiographic measurements taken before and after exercise. This comparison would have provided valuable information on cardiac adaptation to effort in competition $[50,51]$ but was outside the scope of the present study. Hence this topic merits further research.

\section{Conclusion}

In a population of endurance horses aged four to six years, the main changes in cardiac dimensions were observed between age groups and are probably due to ongoing growth, even though the initiation of endurance training may also have had some effect. In particular, LV measurements were related (albeit weakly) to performance parameters, which might reflect the adaption to the initiation of aerobic training. Gender differences were not apparent in our young population, although there were significant differences between breeds. Despite being the smallest horses, purebred Arabians did not have the smallest hearts. Small body size might be an advantage in endurance competitions. However, other factors, such as the heart's dimensions relative to the body dimension, or the capacity of a rapid HR recovery after the race, appear to be important and should be studied in the future. Further longitudinal studies are needed, in particular to validate specific performance indicators for endurance horses. 


\section{Additional files}

Additional file 1: Results of the univariate linear regression analyses, showing the weak influence (expressed as $R^{2}$ ) of the chosen explanatory parameters on left atrial $(L A)$ and great vessel echocardiographic dimensions. (DOCX $16 \mathrm{~kb}$ )

Additional file 2: Results of the univariate linear regression analyses, showing the weak influence (expressed as $R^{2}$ ) of the chosen explanatory parameters on left ventricular (LV) echocardiographic dimensions. (DOCX $16 \mathrm{~kb}$ )

Additional file 3: Results of the multivariate regression analyses, showing the influence of external factors considered as independent variables on left atrial (LA) and great vessel echocardiographic dimensions. (DOCX 29 kb)

Additional file 4: Results of the multivariate regression analyses, showing the influence of external factors considered as independent variables on left ventricular (LV) echocardiographic dimensions. (DOCX $37 \mathrm{~kb}$ )

Additional file 5: Results of the ANOVAs and Student's t-tests reporting p-values for comparisons of morphometric measurements between age groups, genders and breeds groups. (DOCX $17 \mathrm{~kb}$ )

Additional file 6: Results of the ANOVAs and Student's t-tests reporting p-values for comparisons of left atrial (LA) and great vessels dimensions between age groups, genders and breeds groups. (DOCX $18 \mathrm{~kb}$ )

Additional file 7: Results of the ANOVAs and Student's t-tests reporting p-values for comparisons of left ventricular (LV) echocardiographic dimensions between age groups, genders and breeds groups. (DOCX $21 \mathrm{~kb}$ )

\section{Abbreviations}

2DE: Two-dimensional echocardiography; active LA FAC: Active left atrial fractional area change; active:total LA AC: Active-to-total left atrial area change; AIC: Akaike information criterion; ANOVA: Analyse of variance; AOD: The dimension of the aortic (sinus) diameter; BL: Body length; BSA: Body surface area; BWT: Bodyweight; CO: Cardiac output; d: At end-diastole; d-career: Numbers of days since the first recorded official competition; ECG: Electrocardiogram; HR: Heart rate; IVS: Interventricular septal thickness; Km-career: Number of km covered in completion since the first recorded official competition; LA: Left atrium; LAA Left atrial area prior to active contraction, at the onset of the electrocardiographic P-wave; $L A A_{\text {max }}$ : Maximum left atrial area at end-systole, prior to mitral valve

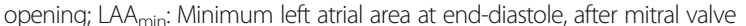
closure; LADa: Left atrial diameter prior to active contraction, at the onset of the electrocardiographic P-wave; LAD max: Maximum left atrial diameter at end-systole, prior to mitral valve opening; $L A D_{\text {min }}$ : Minimum left atrial diameter at end-diastole, after mitral valve closure; LV FAC: Left ventricular fractional area change; LV FS: Left ventricular fractional shortening; LV: Left ventricular; LVFW: Left ventricular free wall thickness; LVIA: Left ventricular internal area; LVID: Left ventricular internal diameter; LVIL: Left ventricular internal length; LVIV: Left ventricular internal volume; $L_{\text {mass: }}$ Left ventricular mass; MWT: Mean wall thickness; PAD: Maximal pulmonary artery diameter; passive LA FAC: Passive left atrial fractional area change; RWT: Relative wall thickness; s: At peak systole; SV: Stroke volume; TC: Thoracic circumference; total LA FAC: Total left atrial fractional area change; WH: Withers height

\section{Acknowledgements}

We would like to acknowledge all the horse owners, riders and trainers as well as collaborators of the project GenEndurance and the Société Hippique Française for making this study possible. Further, we thank Dr Neus Palau Barreda for her valuable assistance during data analysis.

\section{Funding}

The study was funded by the French National Studs (IFCE), the Eperon Funds, the French Arabian Horse Association (ACA), the Institut National de Recherche Agronomique (INRA) and the Ecole Nationale Vétérinaire d'Alfort (ENVA).

\section{Availability of data and materials}

All data supporting the findings for the present study are contained within the manuscript and the additional files mentioned previously.

\section{Authors' contributions}

DST and AG recorded the echocardiographic views, DST, DM and GH performed echocardiographic measurements and some of the statistical analyses, DDH advised on and performed the statistical analyses, and DST, $\mathrm{CR}$ and EB conceived, designed and coordinated the study and drafted the manuscript. All authors read and approved the final manuscript.

\section{Authors' information}

The current address of the corresponding author (DST) is University of Copenhagen, Faculty of Health and Medical Sciences, Department of Veterinary Clinical and Animal Science, Dyrlægevej 100, 1870 Frederiksberg C, Denmark. The current address of GH is Selas Hunaudaye Vétérinaires, 5 PA Carrefour du Penthièvre, 22640 Plestan, France.

Parts of this study have been presented as posters at the 9th ICEPP in

Chester (2014) and at the 7th ECEIM congress in Prague (2014).

\section{Competing interest}

The authors declare that they have no competing interests.

\section{Consent for publication}

Not applicable.

\section{Ethics approval and consent to participate}

The study design was approved by the local institutional Ethical Committee (ComEthANSES/ENVA/UPEC, Maisons-Alfort, France; approval number: 12/07/ 11-1). All owners provided their written, informed consent to participation before the horses underwent any study procedures and were told that they could withdraw their horse from the study at any time.

\section{Author details}

${ }^{1}$ CIRALE-Hippolia, Médecine Sportive, RD 674, F-14430 Goustranville, France. ${ }^{2}$ Université Paris-Est, Ecole Nationale Vétérinaire d'Alfort, 7 avenue du Général de Gaulle, F-94704 Maisons-Alfort, France. ${ }^{3}$ Office Fédéral de la Statistique, Espace de I'Europe 10, CH-2010 Neuchâtel, Switzerland. ${ }^{4}$ INRA, GABI-UMR1313, 78350 Jouy-en-Josas, France.

Received: 18 January 2016 Accepted: 31 August 2016 Published online: 10 October 2016

\section{References}

1. Pluim BM, Zwinderman AH, van der Laarse A, van der Wall EE. The athlete's heart. A meta-analysis of cardiac structure and function. Circulation. 2000;101(3):336-44.

2. D'Andrea A, Limongelli G, Caso P, Sarubbi B, Della Pietra A, Brancaccio P, et al. Association between left ventricular structure and cardiac performance during effort in two morphological forms of athlete's heart. Int J Cardiol. 2002;86(2-3):177-84.

3. Young LE. Cardiac response to training in 2-year-old Thoroughbreds: an echocardiographic study. Equine Vet J Suppl. 1999;30:195-8.

4. Young LE, Rogers K, Wood JL. Left ventricular size and systolic function in Thoroughbred racehorses and their relationships to race performance. J Appl Physiol (1985). 2005;99(4):1278-85.

5. Buhl R, Ersboll AK, Eriksen L, Koch J. Changes over time in echocardiographic measurements in young Standardbred racehorses undergoing training and racing and association with racing performance. J Am Vet Med Assoc. 2005;226(11):1881-7.

6. Buhl R, Ersboll AK. Echocardiographic evaluation of changes in left ventricular size and valvular regurgitation associated with physical training during and after maturity in Standardbred trotters. J Am Vet Med Assoc. 2012;240(2):205-12.

7. Sleeper MM, Durando MM, Holbrook TC, Payton ME, Birks EK. Comparison of echocardiographic measurements in elite and nonelite Arabian endurance horses. Am J Vet Res. 2014;75(10):893-8.

8. Young LE, Rogers $K$, Wood JL. Heart murmurs and valvular regurgitation in thoroughbred racehorses: epidemiology and associations with athletic performance. J Vet Intern Med. 2008;22(2):418-26.

9. Young LE, Wood JL. Effect of age and training on murmurs of atrioventricular valvular regurgitation in young thoroughbreds. Equine Vet J. 2000;32(3):195-9. 
10. Buhl R, Ersboll AK, Eriksen L, Koch J. Use of color Doppler echocardiography to assess the development of valvular regurgitation in Standardbred trotters. J Am Vet Med Assoc. 2005;227(10):1630-5.

11. Young LE, Marlin DJ, Deaton C, Brown-Feltner H, Roberts CA, Wood JL. Heart size estimated by echocardiography correlates with maximal oxygen uptake. Equine Vet J Suppl. 2002;34:467-71.

12. Sampson SN, Tucker RL, Bayly WM. Relationship between VO2max, heart score and echocardiographic measurements obtained at rest and immediately following maximal exercise in thoroughbred horses. Equine Vet J Suppl. 1999;30:190-4.

13. Seder JA, Vickery III CE, Miller PM. The relationship of selected twodimensional echocardiographic measurements to the racing performance of 5431 yearlings and 2003 two-year-old Thoroughbred racehorses. J Equine Vet Sci. 2003;23(4):149-67.

14. Leadon D, McAllister H, Mullins E, Osborne M. Electrocardiographic and echocardiographic measurements and their relationships in Thoroughbred yearlings to subsequent performance. Equine Exp Physiol. 1991;3:22-9.

15. Zucca E, Ferrucci F, Croci C, Di Fabio V, Zaninelli M, Ferro E. Echocardiographic measurements of cardiac dimensions in normal Standardbred racehorses. J Vet Cardiol. 2008;10(1):45-51.

16. Al-Haidar A, Farnir F, Deleuze S, Sandersen CF, Leroux AA, Borde L, et al. Effect of breed, sex, age and body weight on echocardiographic measurements in the equine species. Res Vet Sci. 2013;95(1):255-60.

17. Gehlen H, Haubold A, Stadler P. Reference values for echocardiographic parameters of trained and untrained Icelandic horses. Dtsch Tierarztl Wochenschr. 2007;114(10):374-7.

18. Kocher A, Staniar WB. The pattern of thoroughbred growth is affected by a foal's birthdate. Livest Sci. 2013;154:204-14.

19. Valette JP, Robert C, Denoix JM. Use of linear and non-linear functions to describe the growth of young sport- and race-horses born in Normandy. Animal. 2008;2(4):560-5.

20. Fradinho MJ, Bessa RJB, Ferreira-Dias G, Caldeira RM. Growth and development of the Lusitano horse managed on grazing systems. Livest Sci. 2016:186:22-28.doi:10.1016/j.livsci.2015.06.006.

21. Heugebaert S, Garcia-Launay F, Trillaud-Gey C, Dubroeucq HH, Arnaud G, Bigot $\mathrm{G}$, et al. Modélisation de la croissance des poulains : première étape vers de nouvelles recommandations alimentaires. In: 36ème Journee de la Recherche Equine. Saumur: Institut français du cheval et de l'équitation; 2010. p. 61-68

22. Brown DJ, Rush JE, MacGregor J, Ross Jr JN, Brewer B, Rand WM. M-mode echocardiographic ratio indices in normal dogs, cats, and horses: a novel quantitative method. J Vet Intern Med. 2003;17(5):653-62

23. FFE Compet [https://ffecompet.ffe.com/cheval]

24. Grenacher PA, Schwarzwald CC. Assessment of left ventricular size and function in horses using anatomical M-mode echocardiography. J Vet Cardiol. 2010;12(2):111-21.

25. Schwarzwald CC, Schober KE, Bonagura JD. Methods and reliability of echocardiographic assessment of left atrial size and mechanical function in horses. Am J Vet Res. 2007:68:735-47.

26. Reef VB. Cardiovascular ultrasonography. In: Reef VB, editor. Equine diagnostic ultrasound. Philadelphia: W.B. Saunders; 1998. p. 215-72.

27. Schefer KD, Bitschnau C, Weishaupt MA, Schwarzwald CC. Quantitative analysis of stress echocardiograms in healthy horses with 2-dimensional (2D) echocardiography, anatomical M-mode, tissue Doppler imaging, and 2D speckle tracking. J Vet Intern Med. 2010;24(4):918-31.

28. McConachie E, Barton MH, Rapoport G, Giguere S. Doppler and volumetric echocardiographic methods for cardiac output measurement in standing adult horses. J Vet Intern Med. 2013;27(2):324-30.

29. Lawrence L, Jackson S, Kline K, Moser L, Powell D, Biel M. Observations on body weight and condition of horses in a 150-mile endurance ride. J Equine Vet Sci. 1992;12(5):320-4

30. Garlinghouse SE, Burrill MJ. Relationship of body condition score to completion rate during $160 \mathrm{~km}$ endurance races. Equine Vet J Suppl. 1999;30:591-5.

31. Leleu C, Cotrel C. Body composition in young standardbreds in training: relationships to body condition score, physiological and locomotor variables during exercise. Equine Vet J Suppl. 2006;36:98-101.

32. Kearns CF, McKeever KH, Kumagai K, Abe T. Fat-free mass is related to onemile race performance in elite standardbred horses. Vet J. 2002;163(3):260-6.

33. Brownell KD, Steen SN, Wilmore JH. Weight regulation practices in athletes: analysis of metabolic and health effects. Med Sci Sports Exerc. 1987;19(6):546-56
34. Rodriguez NR, Di Marco NM, Langley S. American College of Sports Medicine position stand. Nutrition and athletic performance. Med Sci Sports Exerc. 2009;41(3):709-31.

35. Kearns CF, McKeever KH, Abe T. Overview of horse body composition and muscle architecture: implications for performance. Vet J. 2002;164(3):224-34.

36. Fleck SJ. Body composition of elite American athletes. Am J Sports Med. 1983;11(6):398-403.

37. Al-Haidar A, Leroux AA, Borde L, Deleuze S, Cerri S, Sandersen CF, et al. Relationship between echocardiographic measurements and body size in horses. J Equine Vet Sci. 2013;33(2):107-14.

38. Martin-Rosset W. Growth and development in the equine. In: 2nd European workshop on Equine Nutrition: Dijon. France: Wageningen Academic Publishers; 2005. p. 15-50.

39. Robert C: Veterinary aspects of training and racing endurance horses. In: Hinchcliff KW, Kaneps AJ, Geor RJ, editors. Equine Sports Medicine and Surgery. 2nd edn. Philiadelphia: Saunders Elsevier; 2014. p. 1083-1106.

40. Maron BJ, Pelliccia A. The heart of trained athletes: cardiac remodeling and the risks of sports, including sudden death. Circulation. 2006;114(15):1633-44.

41. Utomi V, Oxborough D, Whyte GP, Somauroo J, Sharma S, Shave R, et al. Systematic review and meta-analysis of training mode, imaging modality and body size influences on the morphology and function of the male athlete's heart. Heart. 2013:99(23):1727-33.

42. Stewart JH, Rose RJ, Barko AM. Echocardiography in foals from birth to three months old. Equine Vet J. 1984;16(4):332-41.

43. Lombard CW, Evans M, Martin L, Tehrani J. Blood pressure, electrocardiogram and echocardiogram measurements in the growing pony foal. Equine Vet J. 1984;16(4):342-7.

44. Rovira S, Munoz A. Two-dimensional- and M-mode echocardiographic measurements and indices of cardiac function in Spanish colts and fillies of different age. J Vet Med Sci. 2009;71(7):957-64.

45. Hunter S, Robson SC. Adaptation of the maternal heart in pregnancy. Br Heart J. 1992;68(6):540-3.

46. Valensise H, Novelli GP, Vasapollo B, Borzi M, Arduini D, Galante A, et al. Maternal cardiac systolic and diastolic function: relationship with uteroplacental resistances. A Doppler and echocardiographic longitudinal study. Ultrasound Obstet Gynecol. 2000;15(6):487-97.

47. Al Haidar A, Farnir F, Deleuze S, Sandersen CF, Amory H. Comparison of the repeatability of echocardiographic measurements from different modes and views in horses of various breeds and sizes. J Equine Vet Sci. 2010;30(6):287-97.

48. dos Santos Michima LE, Latorre SM, de Andrade AFC, Fernandes WR. Bmode and $M$-mode echocardiography of endurance horses raised in São Paulo State Brazil. J Equine Vet Sci. 2004;24(10):451-7.

49. Sampson SN, Jacobson RL, Sande RD, Susumi CJ, Larntz KJ, Tucker RL, et al. Reproducibility and repeatability of M-mode echocardiographic measurements collected from 25 normal horses. J Equine Vet Sci. 1999;19(1):51-7.

50. Bertone JJ, Paull KS, Wingfield WE, Boon JA. M-mode echocardiographs of endurance horses in the recovery phase of long-distance competition. Am J Vet Res. 1987:48(12):1708-12.

51. Amory H, Votion DM, Fraipont A, Goachet AG, Robert C, Farnir F, et al Altered systolic left ventricular function in horses completing a long distance endurance race. Equine Vet J Suppl. 2010;38:216-9.

\section{Submit your next manuscript to BioMed Central and we will help you at every step:}

- We accept pre-submission inquiries

- Our selector tool helps you to find the most relevant journal

- We provide round the clock customer support

- Convenient online submission

- Thorough peer review

- Inclusion in PubMed and all major indexing services

- Maximum visibility for your research

Submit your manuscript at www.biomedcentral.com/submit 